\title{
Cure of Transverse Instabilities by Chromaticity Modulation
}

\author{
T. Nakamura \\ RIKEN-JAERI Spring-8 Project Team \\ Ako-gun, Hyogo JAPAN
}

\section{abstract}

Landau damping by betatron tune spread suppress transverse instabilities. To create this tune spread, we propose a new method; modulating chromaticity with synchrotron frequency.

\section{INTRODUCTION}

To introduce static tune spread inside of a bunch, we propose the modulation of the chromaticity by synchrotron frequency.

In usual cases, The energy modulation by synchrotron motion and chromaticity can modulate the betatron tune. But the phase shift of the betatron motion becomes zero after one synchrotron period. This means that this modulation of the betatron tune does not effectively suppress the coherent motion or transverse instabilities.

But introducing the chromaticity modulation by synchrotron frequency, the electrons whose synchrotron phase are different each other has different betatron tune and this tune difference is static and does not change.

The other scheme which are used in several storage rings to introduce tune spread inside of a bunch are installation of octupole magnets which cause amplitude dependent tune shift or ion trapping phenomena which introduce nonlinear field just inside and neighborhood of a bunch.

In small emittance rings, the strong octupole fields must be required for the octupole magnet scheme and it cause serious reduction of dynamic apertures. And ion-trapping sometimes causes unwanted motion of the beam.

On the other hand, this scheme, modulating chromaticity with synchrotron frequency, requires some fast sextupole magnets of moderate strength but it does not reduce dynamic apertures seriously[1].

\section{CHROMATICITY MODULATION}

The chromaticity $\xi$ which is modulated by the synchrotron frequency $\omega_{\mathrm{S}}$ is

$$
\xi(t)=\xi_{0}+\hat{\xi}_{1} \cos \omega_{s} t
$$

The relative energy $\left(\mathrm{E}_{-} \mathrm{E}_{0}\right) / \mathrm{E}_{0}$ of a synchrotron oscillation of the electrons is

$$
\varepsilon(t)=\hat{\varepsilon} \cos \left(\omega_{s} t+\phi\right) .
$$

The tune of this electron is

$$
\begin{gathered}
v(t)=\xi(t) \varepsilon(t) \\
=\left[\xi_{0}+\hat{\xi}_{1} \cos \omega_{s} t\right] \hat{\varepsilon} \cos \left(\omega_{s} t+\phi\right) \\
=\xi_{0} \hat{\varepsilon} \cos \left(\omega_{s} t+\phi\right)+\frac{1}{2} \hat{\xi}_{1} \hat{\varepsilon} \cos \phi+\frac{1}{2} \hat{\xi}_{1} \hat{\varepsilon} \cos \left(2 \omega_{s} t+\phi\right) .
\end{gathered}
$$

The second term in equation (3) shows it is the static tune spread and it depends on the phase of the synchrotron motion.

If the distribution of the electron in the phase space of the synchrotron motion is Gaussian;

$$
f(\hat{\varepsilon}, \phi) d \hat{\varepsilon} d \phi=\frac{1}{2 \pi \sigma_{\varepsilon}{ }^{2}} e^{-\frac{\hat{\varepsilon}^{2}}{2 \sigma_{\varepsilon}{ }^{2}}} \hat{\varepsilon} d \hat{\varepsilon} d \phi,
$$

then, the r.m.s. of this static tune spread is

$$
\sigma_{v}=\sqrt{\iint\left(\frac{1}{2} \hat{\xi}_{1} \hat{\varepsilon} \cos \phi\right)^{2} f(\hat{\varepsilon}, \phi) d \hat{\varepsilon} d \phi}=\frac{1}{2} \hat{\xi}_{1} \sigma_{\varepsilon}
$$

. In the SPring- 8 storage ring, $\sigma_{\varepsilon}$ is $1 \times 10^{-3}$ and revolution frequency $\mathrm{f}_{0}$ is $208 \mathrm{kHz}$. If $\xi_{1}$ is set to 1 , then we get $\sigma_{V}=$ $0.5 \times 10^{-3}$. and the damping time $\tau_{L}$ by the Landau damping with this tune spread is $\tau_{L} \sim 1 /\left(2 \pi f_{0} \sigma_{V}\right)=$ $1 /\left(2 \pi \times 208 \mathrm{kHz} \times 0.5 \times 10^{-3}\right)=2 \mathrm{~ms}$. This damping time is faster than the radiation damping time, $8 \mathrm{~ms}$. Figure 1,2,3 and 4 are the result of the tracking simulation of this damping.

Figure 5 is the result of the simulation of multi-bunch instabilities caused by cavity higher-mode transverse impedance. The preliminary study by the simulations for single-bunch instabilities also shows the effectiveness of this scheme to suppress instabilities.

\section{CONCLUSION}

The chromaticity modulation with synchrotron frequency is shown to be effective to create Landau damping and suppress transverse instabilities.

\section{ACKNOWLEDGMENT}

The author thanks to H. Tanaka and the people of the SPring-8 beam physics group for the fruitful discussion and suggestion from the point of view of the transverse single particle dynamics.

\section{REFERENCES}

[1] H. Tanaka, SPring-8, Private communication 


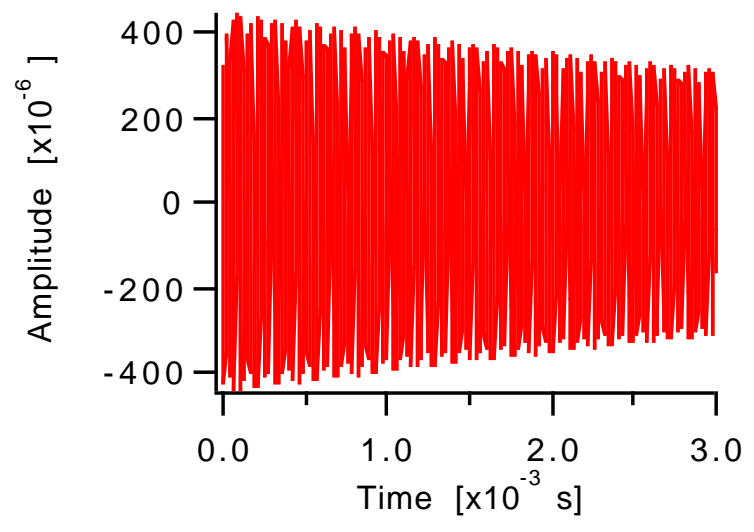

Figure 1

Betatron oscillation without chromaticity modulation. $\xi_{0}=0$ and $\xi_{1}=0$. The radiation damping only $(\tau \sim 8 \mathrm{~ms})$.

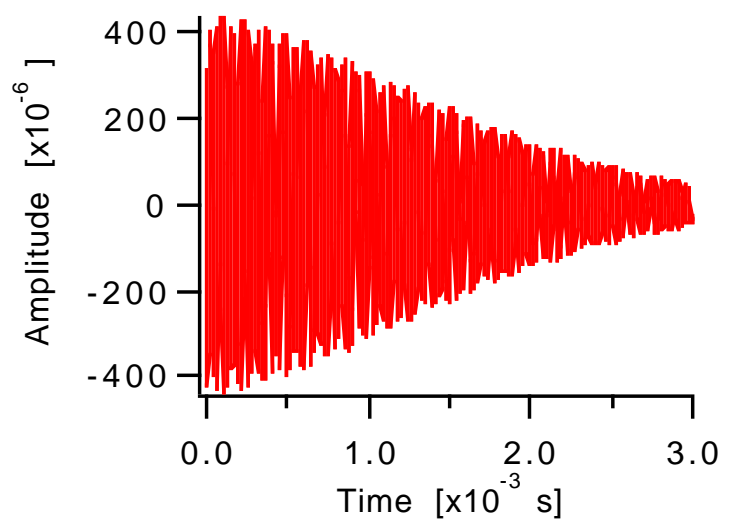

Figure 2

Betatron oscillation with chromaticity modulation. $\xi_{0}=0$ and $\xi_{1}=1$. The faster damping can be seen.

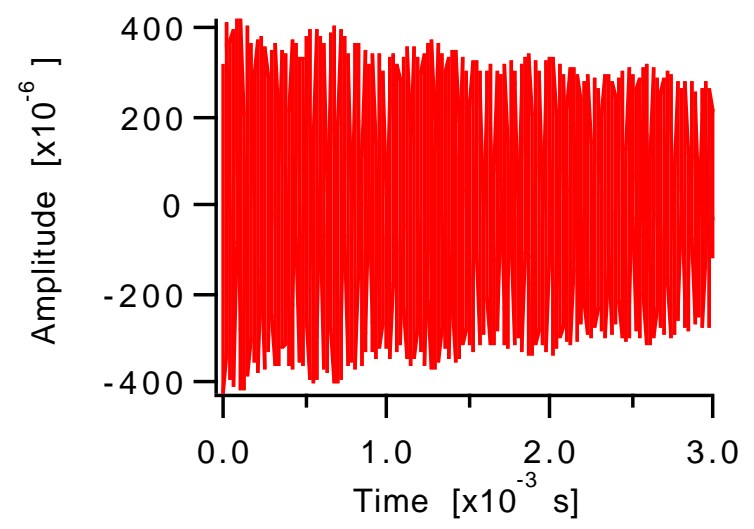

Figure 3

Betatron oscillation without chromaticity modulation. $\xi_{0}=2$ and $\xi_{1}=0$. The radiation damping only $(\tau \sim 8 \mathrm{~ms})$.

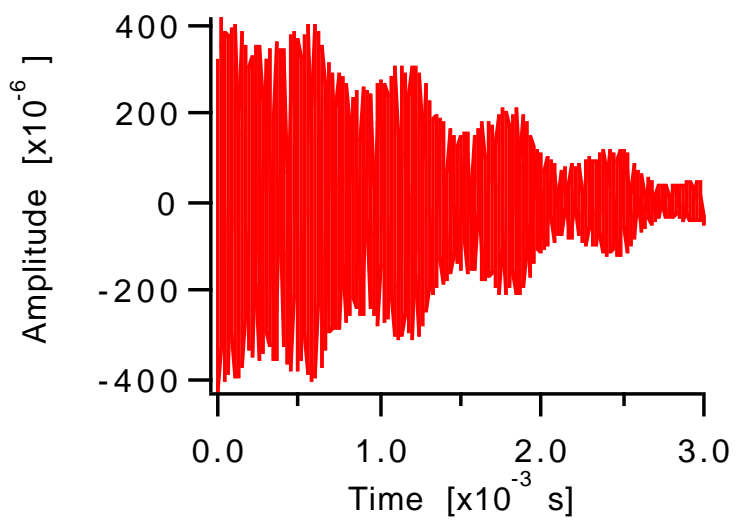

Figure 4

Betatron oscillation with chromaticity modulation. $\xi_{0}=2$ and $\xi_{1}=1$. The faster damping can be seen.

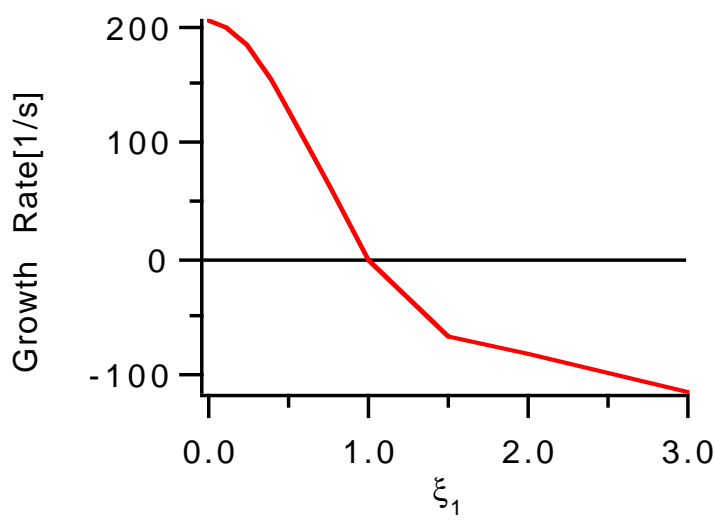

Figure 5

Transverse coupled-instabilities simulation result. This result include radiation damping $(\tau \sim 8 \mathrm{~ms})$. Parameters are of the SPring- 8 storage ring. $\xi_{0}=2, \mathrm{E}=8 \mathrm{GeV}, \mathrm{R} / \mathrm{Q}=2000$, $\mathrm{Q}=13000, \mathrm{f}=1 \mathrm{GHz}, \beta \mathrm{cav}=10 \mathrm{~m}$, frev $=208 \times 10^{3} \mathrm{~Hz}, v_{\mathrm{X}}=51.22$, $v_{\mathrm{S}}=0.01$. 